\title{
Crystalline Approximant of Amorphous Fe-Si-B Structures
}

\author{
Akihiko Hirata ${ }^{1,2, *}$ \\ ${ }^{1}$ WPI Advanced Institute for Materials Research, Tohoku University, Sendai 980-8577, Japan \\ ${ }^{2}$ Mathematics for Advanced Materials-OIL, AIST-Tohoku University, Sendai 980-8577, Japan
}

\begin{abstract}
We have examined the local atomic configurations of amorphous Fe-Si-B alloys by means of electron diffraction pair-distribution-function analysis and compared them with those of a metastable sigma phase formed during the crystallization process. We found a great structural similarity, not only at short range but also at medium range up to $1.0 \mathrm{~nm}$, between amorphous Fe-Si-B and sigma phase structures. The results imply that the sigma phase structure can be regarded as a good crystalline approximant for the glass structures in this alloy system. The connections of coordination polyhedra in the sigma phase structure were also discussed. [doi:10.2320/matertrans.MD201711]
\end{abstract}

(Received November 21, 2017; Accepted January 12, 2018; Published March 2, 2018)

Keywords: amorphous alloys, electron diffraction, local structures

\section{Introduction}

The atomic configurations of amorphous alloys have long been debated through experimental and theoretical approaches. ${ }^{1-8)}$ A dense random packing description for liquids proposed by Bernal in an early stage has been recognized as a good prototypical model for amorphous alloys. ${ }^{9,10)}$ Currently, it is widely accepted that nearestneighbor atomic environments of amorphous alloys build up coordination polyhedra with coordination numbers from approximately 8 to 16 , which are largely dependent on the atomic size differences. ${ }^{6-8)}$ It should be noted that the coordination numbers in amorphous alloys are much larger than those in many other amorphous materials, such as oxides, chalcogenides, and polymers. The coordination polyhedra in amorphous alloys have been directly observed by means of a subnanometer-scale electron diffraction technique. ${ }^{11,12)}$ More importantly, it is necessary to unravel how coordination polyhedra can fill an entire three-dimensional space to realize the overall densely packed structure.

For short-range scale configurations, the structural similarity between amorphous and crystal structures has frequently been pointed out based on coordination numbers, as well as pair-distribution function analyses. ${ }^{13,14)}$ However, it is still challenging to find good crystalline approximants that reproduce amorphous alloy structures beyond the nearest-neighbor scale, as is the case for quasicrystals. ${ }^{15}$ ) Moreover, the fact that there remain unsolved crystal structures of metastable phases appearing in amorphous alloy systems considerably restricts the finding. In this study, we identified a metastable sigma phase crystal appearing in the crystallization process of the $\mathrm{Fe}-\mathrm{Si}-\mathrm{B}$ alloy system and demonstrated the structural similarity between the crystal and amorphous structures up to $1.0 \mathrm{~nm}$.

\section{Experimental Procedure}

Commercial amorphous $\mathrm{Fe}-\mathrm{Si}$-B ribbons (METGLAS $2605 \mathrm{~S}-2\left(\mathrm{Fe}_{78} \mathrm{Si}_{9} \mathrm{~B}_{13}\right)$ and $\left.2605 \mathrm{~S}-3\left(\mathrm{Fe}_{79} \mathrm{Si}_{5} \mathrm{~B}_{16}\right)\right)$ were used for transmission electron microscopy (TEM) observations. To

*Corresponding author, E-mail: hirata@wpi-aimr.tohoku.ac.jp observe a partially crystallized microstructure of $\mathrm{Fe}_{78} \mathrm{Si}_{9} \mathrm{~B}_{13}$, the ribbon specimen was annealed isothermally at $763 \mathrm{~K}$ for $0.5 \mathrm{~h}$ in a vacuum furnace. Specimens for TEM observation were prepared by electropolishing (aceticperchloric acid) at $300 \mathrm{~K}$. The specimens were finally polished by using ion-milling (GATAN PIPS) with a low voltage $(2.5 \mathrm{kV})$ and a glancing angle of $4^{\circ}$. Selected area electron diffraction (SAED) patterns and bright-field images were taken by a $200 \mathrm{kV}$ TEM (LEO-922D) equipped with an omega-type energy filter. SAED patterns were recorded using an imaging plate system (DITABIS Micron). A detailed procedure for analyzing electron diffraction intensity is described elsewhere. ${ }^{16)}$

\section{Results and Discussion}

Before analyzing the amorphous structures, we examined a crystalline product of $\mathrm{Fe}_{78} \mathrm{Si}_{9} \mathrm{~B}_{13}$ in the partially crystallized sample. Figure 1(a) shows a bright-field image of the crystalline precipitate embedded in the amorphous matrix, together with a corresponding SAED pattern in Fig. 1(b). The halo rings and diffraction spots in Fig. 1(b) originated from the featureless bright matrix and dark region in the image, respectively. To identify the crystal structure of the dark region, we calculated a diffraction pattern of the sigma phase (prototype: $\sigma$-CrFe, tP30), ${ }^{17)}$ which is a Frank-Kasper phase, ${ }^{18-20)}$ because a formation of the sigma phase in Fe$\mathrm{Si}-\mathrm{B}$ systems has already been reported in the literature. ${ }^{21)}$ The [001] projection of the atomic configuration of the sigma phase is depicted in Fig. 1(c). Because the calculated diffraction pattern in Fig. 1(d) is consistent with the experimental pattern in Fig. 1(b), we concluded that the sigma phase is also formed in the present alloy system $\left(\mathrm{Fe}_{78} \mathrm{Si}_{9} \mathrm{~B}_{13}\right)$.

Figure 2 shows reduced interference functions $Q \mathrm{i}(Q)$ obtained from amorphous $\mathrm{Fe}_{78} \mathrm{Si}_{9} \mathrm{~B}_{13}$ and $\mathrm{Fe}_{79} \mathrm{Si}_{5} \mathrm{~B}_{16}$ specimens. The $Q \mathrm{i}(Q)$ profiles were calculated from energyfiltered SAED patterns, as shown in the inset. The features of these profiles are typically found in metal-metalloid amorphous alloys. In our measurement, intensity oscillations up to $180 \mathrm{~nm}^{-1}$ are clearly observed for both of the profiles. This makes it possible to see detailed real-space information 


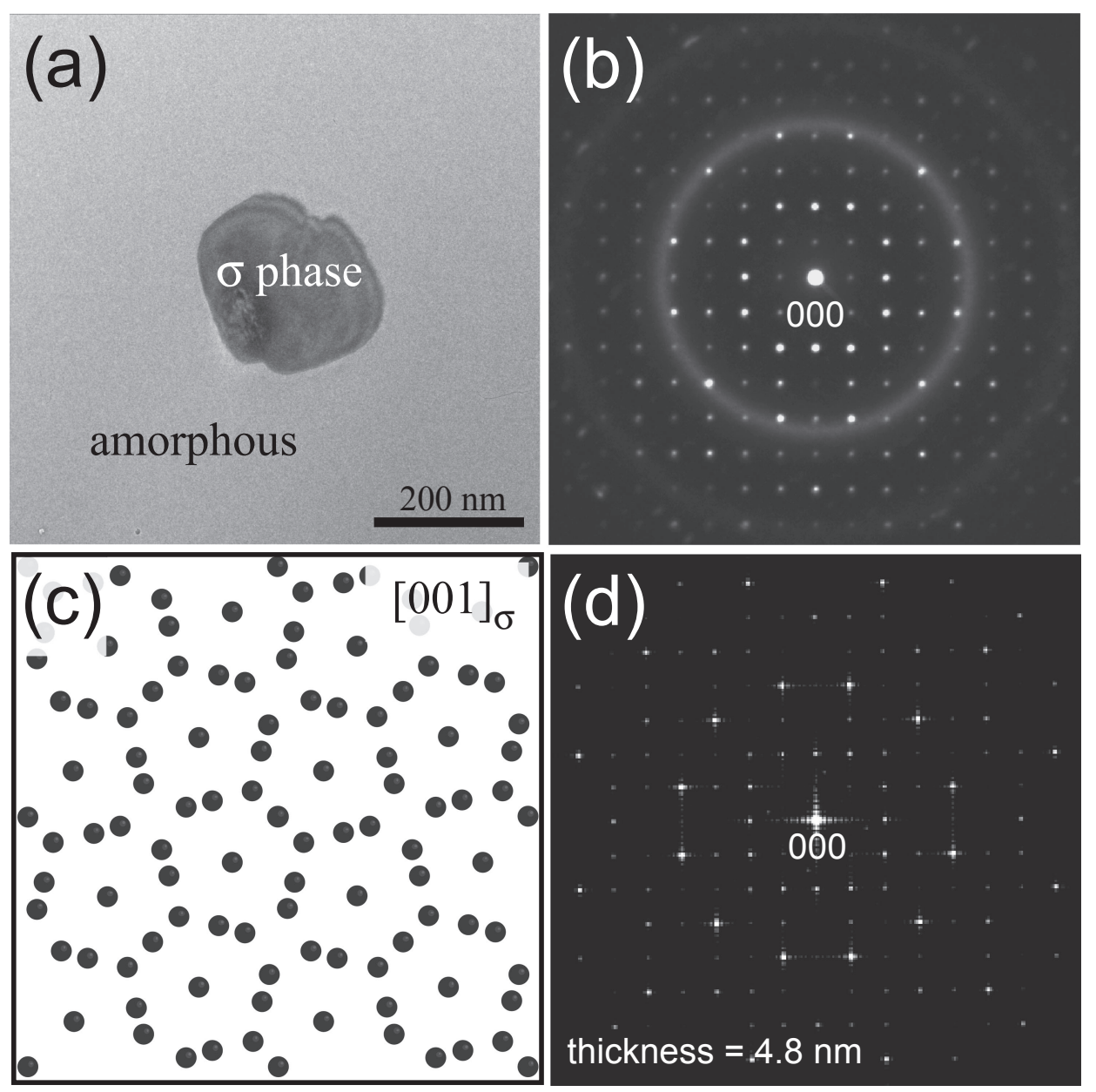

Fig. 1 (a) Bright-field image of $\mathrm{Fe}_{78} \mathrm{Si}_{9} \mathrm{~B}_{13}$ annealed at $763 \mathrm{~K}$ for $0.5 \mathrm{~h}$, (b) Selected electron diffraction pattern obtained from the region of (a), (c) [001] projection of the sigma phase structure, and (d) Simulated electron diffraction pattern of the sigma phase structure. A thickness of the structure model was $4.8 \mathrm{~nm}$.

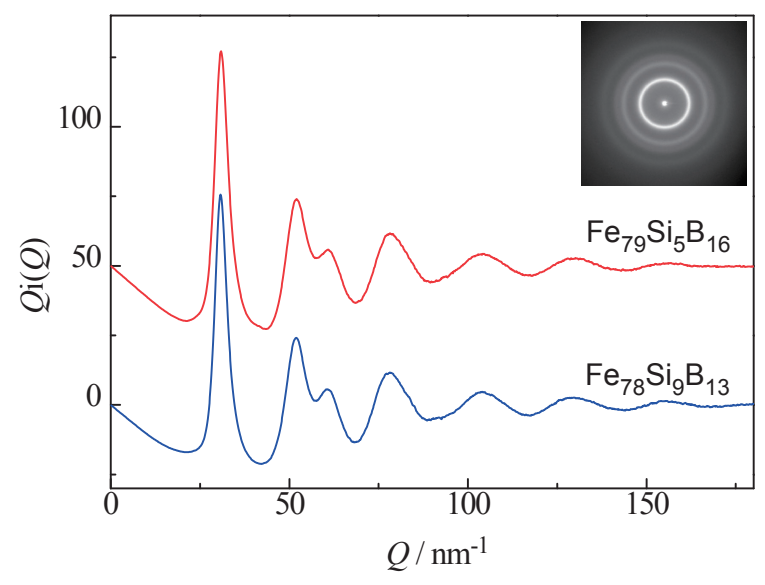

Fig. 2 Reduced interference function $Q \mathrm{i}(Q)$ profiles obtained from asformed amorphous $\mathrm{Fe}_{78} \mathrm{Si}_{9} \mathrm{~B}_{13}$ and $\mathrm{Fe}_{79} \mathrm{Si}_{5} \mathrm{~B}_{16}$ specimens. An example of energy-filtered electron diffraction patterns used for $Q \mathrm{i}(Q)$ is shown in the inset. The diffraction pattern was taken from a circular region with a diameter of $100 \mathrm{~nm}$.

through a Fourier transform operation. Figure 3 shows pairdistribution functions $\mathrm{g}(\mathrm{r})$ for $\mathrm{Fe}_{78} \mathrm{Si}_{9} \mathrm{~B}_{13}$ and $\mathrm{Fe}_{79} \mathrm{Si}_{5} \mathrm{~B}_{16}$, transformed from the $Q \mathrm{i}(Q)$ profiles shown in Fig. 2. Interatomic correlations in real space up to $1.0 \mathrm{~nm}$ are clearly seen in both of the profiles, together with a distinct 2 nd-peak

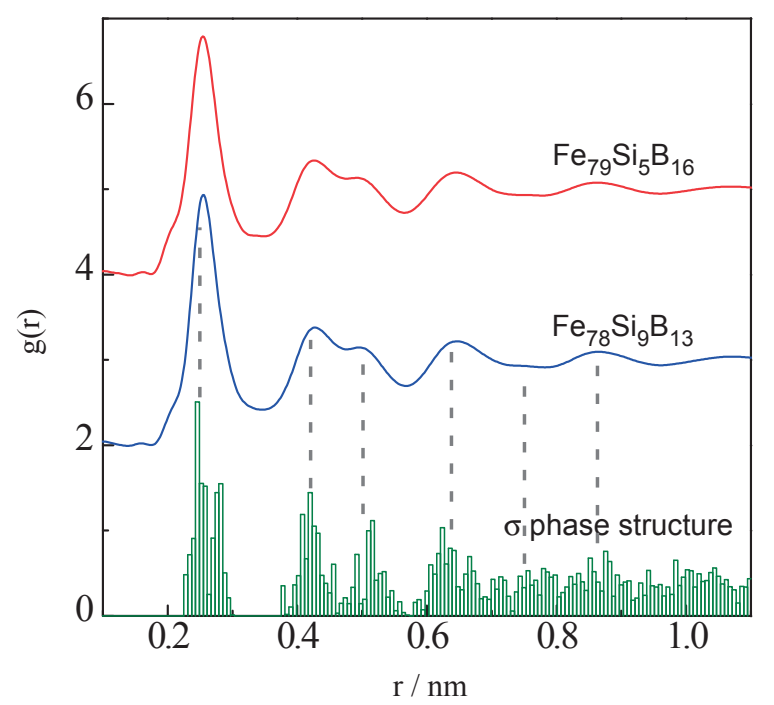

Fig. 3 Pair distribution function $\mathrm{g}(\mathrm{r})$ profiles for amorphous $\mathrm{Fe}_{78} \mathrm{Si}_{9} \mathrm{~B}_{13}$ and $\mathrm{Fe}_{79} \mathrm{Si}_{5} \mathrm{~B}_{16}$ calculated by $Q \mathrm{i}(Q)$ profiles shown in Fig. 2. The $\mathrm{g}(\mathrm{r})$ profile calculated from the sigma phase structure is also shown.

split around $0.45 \mathrm{~nm}$. We also calculated a $\mathrm{g}(\mathrm{r})$ profile from the sigma phase structure appearing in the crystallization process (see Fig. 1) for comparison. Interestingly, it was found that the sigma phase structure accurately reproduces 
(a)

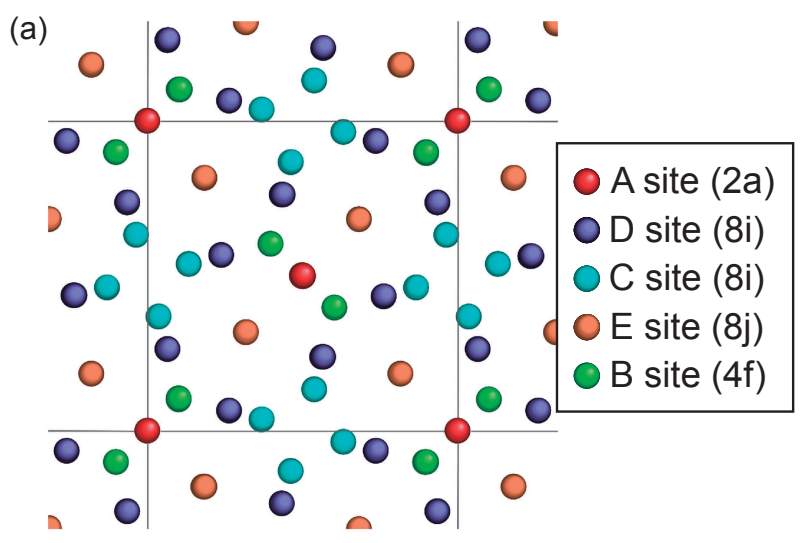

(b)

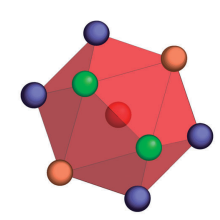

CN12 (A site)

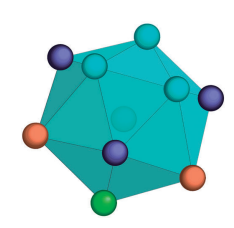

CN14 (C site)

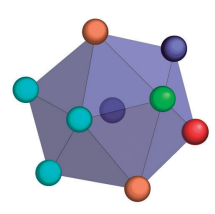

CN12 (D site)

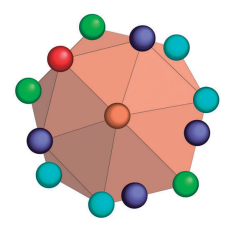

CN14 (E site)

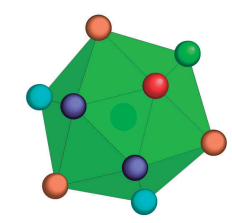

CN15 (B site)

Fig. 4 (a) [001] projection of the sigma phase structure. Crystallographically different atomic sites (A to E sites) are denoted with different colors. (b) Five coordination polyhedra whose central atoms are positioned at each atomic site.

not only the peak-top positions, but also wide the dispersions found in the amorphous profiles. It should be noted that simple crystals such as fcc, hep, and bcc never show a broad $\mathrm{g}(\mathrm{r})$ profile with such dispersions.

On the basis of the similarity between the amorphous and sigma phase structures as shown above, we discuss mediumrange structures of amorphous Fe-Si-B samples, particularly relevant to coordination polyhedra. Figure 4(a) illustrates a [001] projection of the sigma phase structure, where five atomic sites are depicted with different colors. As shown in Fig. 4(b), the A, D, C, E, and B sites, which are named according to reference 20 , form polyhedra with coordination numbers of $12,12,14,14$, and 15 , respectively. We should emphasize that all the atomic sites can be the central atom of coordination polyhedra. This implies that all the coordination polyhedra are interconnected by sharing several atoms. Additionally, atoms surrounding the D, E, and B sites can be the central atom of all the other coordination polyhedra. From the structural similarity, as shown in Fig. 3, the medium-range structures in amorphous alloys should be in a similar situation to those in the sigma phase.

Although the polyhedra with coordination number 12 in the sigma phase essentially have an icosahedral symmetry, it is heavily distorted compared to the ideal icosahedron. It is understood that the distortion leads to the wide dispersion of the $\mathrm{g}(\mathrm{r})$ profile in Fig. 3(c). This is quite similar to the

situation in an amorphous alloy structure, as we observed before. ${ }^{12)}$ The distortion of polyhedra plays a significant role in filling a three-dimensional space without large voids for both polyhedron-based crystals, including the sigma phase, as well as amorphous alloys.

We finally discuss the role of $\mathrm{Si}$ atoms in amorphous and sigma phase structures. Unfortunately, it is still not clear which sites are occupied by $\mathrm{Si}$ atoms in the sigma phase structure. However, we can infer that $\mathrm{Si}$ atoms significantly contribute to the stability of the sigma phase, because this phase was not found in the binary Fe-B alloys. For the amorphous Fe-Si-B, moreover, the previous study reported that the bond length of $\mathrm{Fe}-\mathrm{Si}$ is much longer than that of $\mathrm{Fe}-\mathrm{B}$ and close to that of $\mathrm{Fe}-\mathrm{Fe}{ }^{22)}$ This implies that $\mathrm{Si}$ atoms are replaceable with Fe rather than $\mathrm{B}$ in amorphous structures and also probably in crystal structures. Because the metalloid (only B) concentrations for $\mathrm{Fe}_{78} \mathrm{Si}_{9} \mathrm{~B}_{13}$ and $\mathrm{Fe}_{79} \mathrm{Si}_{5} \mathrm{~B}_{16}$ are quite low, though $\mathrm{Fe}-\mathrm{Si}-\mathrm{B}$ is a metal-metalloid type, the network of coordination polyhedra with relatively large coordination numbers found in the sigma phase well reproduces the overall features of amorphous structures.

\section{Conclusion}

We found that the sigma phase structure, which contains coordination polyhedra, is a good crystalline approximant of the amorphous structures in Fe-Si-B alloy systems. An understanding of the crystalline approximants, where coordination polyhedra are interconnected, should be very helpful for exploring the amorphous structures beyond the nearest-neighbor scale. In the near future, we will search for more crystalline approximants, which may be metastable phases rather than equilibrium phases, not only for metalmetalloid systems, but also for metal-metal systems.

\section{Acknowledgements}

This work was partially sponsored by Fusion Research Funds from the "World Premier International Research Center Initiative for Atoms, Molecules, and Materials" program of the MEXT of Japan, JSPS KAKENHI (grant numbers JP17H01325 and JP26310205). The author gratefully acknowledges Prof. Yoshihiko Hirotsu at Osaka University for the development of electron diffraction intensity analysis techniques and the provision of TEM equipments.

\section{REFERENCES}

1) Y.Q. Cheng and E. Ma: Prog. Mater. Sci. 56 (2011) 379-473.

2) E. Ma: Nat. Mater. 14 (2015) 547-552.

3) C.P. Royall and S.R. Williams: Phys. Rep. 560 (2015) 1-75.

4) E. Matsubara and Y. Waseda: Mater. Trans. JIM 36 (1995) 883.

5) Y. Hirotsu, T. Ohkubo and M. Matsushita: Microsc. Res. Tech. 40 (1998) 284.

6) D.B. Miracle: Nat. Mater. 3 (2004) 697-702.

7) D.B. Miracle: Acta Mater. 54 (2006) 4317-4336.

8) H.W. Sheng, W.K. Luo, F.M. Alamgir, J.M. Bai and E. Ma: Nature 439 (2006) 419-425.

9) J.D. Bernal: Nature 17 (1959) 141-147.

10) J.A. Barker, M.R. Hoare and J.L. Finney: Nature 257 (1975) 120-122.

11) A. Hirata, P. Guan, T. Fujita, Y. Hirotsu, A. Inoue, A.R. Yavari, T. Sakurai and M. Chen: Nat. Mater. 10 (2011) 28-33. 
12) A. Hirata, L.J. Kang, T. Fujita, B. Klumov, K. Matsue, M. Kotani, A.R. Yavari and M.W. Chen: Science 341 (2013) 376-379.

13) V.Y. Kazimirov, D. Louca, V. Ponnambalam, S.J. Poon and T. Proffen: Phys. Rev. B 72 (2005) 054207.

14) D.V. Louzguine-Luzgin, A.R. Yavari, G. Vaughan and A. Inoue: Intermetallics 17 (2009) 477-480.

15) V. Elser and C.L. Henley: Phys. Rev. Lett. 55 (1985) 2883.

16) A. Hirata, Y. Hirotsu, T. Ohkubo, T. Hanada and V.Z. Bengus: Phys.
Rev. B 74 (2006) 214206.

17) B.G. Bergman and D.P. Shoemaker: J. Chem. Phys. 19 (1951) 515.

18) F.C. Frank and J.S. Kasper: Acta Crystallogr. 11 (1958) 184-190.

19) F.C. Frank and J.S. Kasper: Acta Crystallogr. 12 (1959) 483-499.

20) A.K. Sinha: Prog. Mater. Sci. 15 (1972) 79-185.

21) N.B. Dyakonova, I.V. Lyasotskii, E.N. Vlasova, D.L. Dyakonov and B.V. Molotilov: Adv. Perform. Mater. 4 (1997) 199-207.

22) Y. Takahara and N. Narita: Mater. Sci. Eng. A 315 (2001) 153-157. 